International Journal of Pure and Applied Mathematics

Volume 88 No. 2 2013, 247-262

ISSN: 1311-8080 (printed version); ISSN: 1314-3395 (on-line version)

url: http://www.ijpam.eu

doi: http://dx.doi.org/10.12732/ijpam.v88i2.8

ijpam.eu

\title{
A MAX-PLUS MODEL FOR GENETIC ALGORITHMS
}

\author{
Anthony Y. Aidoo ${ }^{1}$, Joseph Ackora-Prah ${ }^{2}$, \\ Kwasi Baah Gyamfi ${ }^{3}$, Bonsu Mensah Osei ${ }^{4}$ \\ ${ }^{1}$ Department of Mathematics and Computer Science \\ Eastern Connecticut State University \\ Willimantic, CT 06226, USA \\ ${ }^{2,3}$ Department of Mathematics \\ Kwame Nkrumah University of Science and Technology \\ Kumasi, GHANA \\ ${ }^{4}$ Department of Mathematics and Computer Science \\ Eastern Connecticut State University \\ Willimantic, CT 06226, USA
}

\begin{abstract}
In this paper, a genetic algorithm (GA) reformulation that combines the properties of max-plus algebra is outlined. This theoretical analysis provides a useful framework for the determination the optimal solution of an optimization problem through a fitness function. The model allows us to handle the many properties, concepts and techniques of GA's in a simplified manner.
\end{abstract}

AMS Subject Classification: 15A80, 34H05, 49J35, 49J99

Key Words: max-plus algebra, optimization, genetic algorithm

\section{Introduction}

GA's are utilized to solve a variety of problems in fields such as artificial intelligence in computer science, bioinformatics, manufacturing, and mathematics. However, not all optimization problems, classical or otherwise, can be solved by using GA. Certain optimization problems known as variant problems fall into

Received: July 10, 2013

(C) 2013 Academic Publications, Ltd.

${ }^{\S}$ Correspondence author url: www.acadpubl.eu 
this category. This is because poorly known fitness functions generate inefficient chromosome blocks, despite the fact that only good chromosome blocks crossover. In addition, even though GA explores the optimization search space using the population of search points, it is usually plagued by poor convergence properties [14]. In order to correct this and some of other well known disadvantages of GA's we reformulate the GA scheme using max-plus algebra.

Max-plus algebra has been applied to several fields including optimal control and the solution of homogeneous two-sided systems of equations $[4,3]$ and the solvability concepts for interval systems of linear equations, [11, 13], as well as to systems theory [1]. It becomes imperative to investigate new methods such as provided by max-plus algebra to improve GA's.

\section{Preliminaries}

In max-plus algebra, we consider the algebraic structure made up of the triple $\left(\mathbb{R}_{\max }, \oplus, \otimes\right)$, where $\mathbb{R}_{\max }=\mathbb{R} \cup\{-\infty\}$ and $\oplus, \otimes$ are operations defined by $a \oplus b=\max (a, b)$ and $a \otimes b=a+b$, for $a, b \in \mathbb{R}_{\max }$. The algebraic structure is an idempotent semi-ring whose elements are the usual real numbers together with $-\infty$ which will be denoted by $\varepsilon$ throughout this paper and 0 will be denoted by $e$.

The algebraic structure establishes a duality between probability and optimization based on this algebra. It has been shown that the max operator models the fact that as soon as the preconditions to a task has been fulfilled, the task itself can be fulfilled [2].

Applications of this algebraic structure to optimization requires the solution of the max-plus linear equation in $\mathbb{R}_{\max }^{n \times n}$, whose generalized form can be written as $A \otimes X \oplus b=C \otimes X \oplus d$. This max-plus formulation helps to provide a mathematical framework model for the population dynamics of the GA processes and it also addresses some of the disadvantages of Genetic algorithms.

For matrices and vectors, the max-plus algebra operations are defined as follows. Let $M=1, \ldots, m, N=1, \ldots, n$ and let $A, B, C \in \mathbb{R}_{\max }^{n \times m}$, for $i \in N$ and $j \in M$. Let $A=\left(a_{i j}\right), B=\left(b_{i j}\right)$ and $C=\left(c_{i j}\right)$ be matrices of compatible sizes, then

$$
\begin{aligned}
A \oplus B & =\left(a_{i j} \oplus b_{i j}\right) \\
A \otimes B & =\left(\Sigma_{k}^{\oplus} a_{i k} \otimes b_{k j}\right) \\
& =\max _{k}\left(a_{i k}+b_{k j}\right)
\end{aligned}
$$




$$
\text { and } \begin{aligned}
\alpha \otimes A & =\left(\alpha \otimes a_{i j}\right) \\
& =A \otimes \alpha
\end{aligned}
$$

[7]

In particular, the matrix product $A \otimes B$ is defined for matrices $A \in \mathbb{R}_{\max }^{\operatorname{m} \times p}$, $B \in \mathbb{R}_{\max }^{p \times n}$ as a matrix $C \in \mathbb{R}_{\max }^{m \times n}$ by the formula

$$
C_{i j}=\oplus_{k=1}^{p}\left(a_{i k} \otimes b_{k j}\right)=\max _{k=1, \ldots, p}\left(a_{i k}+b_{k j}\right)
$$

for $i=1, \ldots, m, j=1, \ldots, n[16]$.

Theorem 1. Let $N=1, \ldots, n$. Let $A, B \in \mathbb{R}^{m \times n}$ and let $\left(C_{1}(j, i), C_{2}(j, i) \ldots\right)$ $=C(j, i)=A_{j}-B_{i}$ and denote by $C_{k}(j, i)$ the $k$-th component of $C(j, i), k \in$ $N$. Let $A, B$ be such that for all $i, j, k \in N$ with $j=k \neq i, C_{k}(j, i)>C_{i}(j, i)$. Then the equation $A \otimes x=B \otimes x$ is solvable if and $C$ is definite.

Proof. See for example, [4].

\subsection{One-Sided-Linear-System and Eigenvalue Problems}

We consider the following one-sided linear system and eigenvalue problems and their solutions which are major concepts in max-plus algebra and are adopted for our formulation of GA's. Consider the equations:

$$
A \otimes x=b
$$

$$
A \otimes x=\lambda \otimes x
$$

To solve the systems (2), (3) we define the following:

$$
\begin{aligned}
S(A, b) & =\left\{x \in R^{n} \mid A \otimes x=b\right\} \\
M_{j}(A, b) & =\left\{k \in M \mid\left(a_{k j}-b_{k}\right)=\max _{i=1, \ldots, m}\left(a_{i j}-b_{i}\right)\right\}, \forall j \in N, \\
\overline{x_{j}} & =-\max _{i=1, \ldots, m}\left(a_{i j}-b_{i}\right), \forall j \in N .
\end{aligned}
$$

We note that if $b=\varepsilon$, then $S(A, b)=\left\{x \in R^{n} \mid x_{j}=\varepsilon\right.$ if $\left.A_{j} \neq \varepsilon, j \in N\right\}$ and if $A=\varepsilon$, then we have $S(A, b)=R^{n}$. Let $A=\varepsilon$ and $b \neq \varepsilon$, then $S(A, b)=\emptyset$. Now let $A \neq \varepsilon$ and $b \neq \varepsilon$ and suppose that $b_{k}=\varepsilon \forall k \in M$, then for any $x \in S(A, b), x_{j}=\varepsilon$ if $a_{k j} \neq \varepsilon, j \in N$. This implies that the $k^{t h}$ equation can be removed from the system. Consequently putting $x_{j}=\varepsilon$ for 
columns $A_{j}, a_{k j} \neq \varepsilon$, we can also remove the columns from the system. We can therefore assume that $b$ is finite without loss of generality. If $b$ is finite and $A_{j}=\varepsilon$, then $x_{j} \in x$. Such matrices are called row R-astic (column R-astic). A matrix is called doubly R-astic if it is both row and column R-astic [8]. We can therefore suppose without loss of generality that $A$ is doubly $R$-astic, that is $A$ has no $A_{j}=\varepsilon$ rows and columns, [8]. The following theorem shows that the system (1) has solution if and only if $\bar{x}$ is a solution to the system.

Theorem 2. Let $A \in R^{m \times n}$ be doubly $R$-astic and $b \in R^{m}$. Then $x \in S(A, b)$ if and only if

(i) $x \leq \bar{x}$ and

(ii) $\bigcup_{j \in N_{x}} M_{j}(A, b)=M$ where $N_{x}=\left\{j \in N \mid x_{j}=\overline{x_{j}}\right\}$

Corollary (1) below gives the the condition for the solution of the system $A \otimes x=$ $b$ and Corollary (2) provides the condition for uniqueness of solution of the systems.

Corollary 1. Let $A \in R^{m \times n}$ be doubly $R$-astic and $b \in R^{m}$. Then the following 3 statements are equivalent:

(i) $S(A, b) \neq \emptyset$

(ii) $\bar{x} \in S(A, b)$

(iii) $\bigcup_{j \in N} M_{j}(A, b)=M$

Corollary 2. Let $A \in R^{m \times n}$ be doubly $R$-astic and $b \in R^{m}$. Then $S(A, b)=\{\bar{x}\}$ if and only if:

(i) $\bigcup_{j \in N} M_{j}(A, b)=M$ and

(ii) $\bigcup_{j \in N^{\prime}} M_{j}(A, b) \neq M$ for any $N^{\prime} \subseteq N, N^{\prime} \neq N$

The maximum cycle mean of a matrix is of fundamental importance in maxplus algebra since it is the greatest max-algebraic eigenvalue for any square matrix. The max-plus eigenvalue-eigenvector problem $A \otimes x=\lambda \otimes x$ has a unique value of $\lambda=\lambda(A)$, called the eigenvalue of $A$ to which there is an $x \in \mathbb{R}_{\text {max }}^{n}$ satisfying the equation $A \otimes x=\lambda \otimes x$. The unique eigenvalue is the maximum cycle mean of $A$ that is

$$
\lambda(A)=\max _{\sigma} \frac{\omega(A, \sigma)}{l(\sigma)}
$$

where $\sigma=\left(i_{1}, \ldots, i_{k}\right)$ denotes an elementary cycle (that is a cycle with no repeated node except the first and the last one) in $D_{A}, \omega(A, \sigma)=a_{i_{1} i_{2}}+\ldots+a_{i_{k} i_{1}}$ is the weight of $\sigma$ and $l(\sigma)=k$ is the length of $\sigma$. The maximization is taken over elementary cycles of all lengths in $D_{A}$, including the loops. The computation of the maximum cycle mean is difficult since the number of cycles is very large 
in general. The best known method currently is Karp's algorithm [10] which is based on the following theorem:

Theorem 3. If $A=\left(a_{i j}\right) \in \mathbb{R}_{\max }^{n \times n}$ is irreducible then

$$
\lambda(A)=\max _{j \in N} \min _{k \in N} \frac{F_{n+1}(j)-F_{k}(j)}{n+1-j}
$$

where $F_{k}(j)$ is the maximum weight of an $s-j$ path of length $k$.

Now let

$$
\begin{array}{r}
\Gamma(A)=A \otimes A^{2} \otimes A^{3} \otimes \ldots \\
\triangle(A)=I \oplus \Gamma(A)=I \oplus A \oplus A^{2} \oplus A^{3} \oplus \ldots
\end{array}
$$

The matrix $\Gamma(A)$ is the weak transitive closure of $A$ if the series in (5) converges, and $\triangle(A)$ is the strong transitive closure of $A$. We use these matrices to describe all non-trivial solutions (if any) to the max-plus equation:

$$
A \otimes x=x
$$

in the case of $\Gamma(A)$ and all finite solutions to

$$
A \otimes x \leq \lambda \otimes x
$$

in the case of $\triangle(A)$. If $\lambda(A) \leq 0$ then $\Gamma(A)=A \oplus A^{2} \oplus A^{3} \oplus \ldots \oplus A^{k}$ for every $k \geq n$. If $A$ is irreducible and $n>1$ then $\Gamma(A)$ is finite. Hence if $\lambda(A) \leq 0$ then it implies $A^{k} \leq A \oplus A^{2} \oplus A^{3} \oplus \ldots \oplus A^{n}$ for every $k \geq 1$ and therefore $\Gamma(A)$, for any matrix with $\lambda(A) \leq 0$ exists, and is equal to $A \oplus A^{2} \oplus A^{3} \oplus \ldots \oplus A^{n}$.

Again if $\lambda(A) \leq 0$ we have $\triangle(A)=I \oplus \Gamma(A)=I \oplus A \oplus A^{2} \oplus A^{3} \oplus \ldots \oplus A^{k}$ for every $k \geq n-1$ which implies that $A \otimes \triangle(A)=A \otimes\left(I \oplus A \oplus A^{2} \oplus A^{3} \oplus \ldots \oplus A^{k-1}\right)=$ $A \oplus A^{2} \oplus A^{3} \oplus \ldots \oplus A^{n}=\Gamma(A) \leq \triangle(A)$, that is every column of $\triangle(A)$ is a solution to $A \otimes x \leq x$. Similarly if $\lambda(A) \leq 0$ we have $A \otimes \Gamma(A)=$ $A \otimes\left(A \oplus A^{2} \oplus A^{3} \oplus \ldots \oplus A^{n}\right)=A^{2} \oplus A^{3} \oplus \ldots \oplus A^{n+1} \leq A \oplus A^{2} \oplus A^{3} \oplus \ldots \oplus A^{n+1}=\Gamma(A)$ which also implies that every column of $\Gamma(A)$ is also a solution to $A \otimes x \leq x$. If $\lambda(A)=0$, then at least one column of $\Gamma(A)$ is a solution to $A \otimes x=x$. We note that $A \otimes x \leq \lambda \otimes x$ has a finite solution if and only if $\lambda(A) \leq \lambda$.

The conjugate of $A=a_{i j} \in R^{m \times n}$ is $A^{*}=a_{i j}^{*} \in R^{m \times n} \forall i \in M, j \in N$, where $a_{i j}^{*}=\left(a_{j i}\right)^{*}$. The importance of conjugation in solving the system (1) is shown by the following theorem:

Theorem 4. If $A \in R^{m \times n}, b \in R^{m}$ and $x \in R^{n}$ then $A \otimes x \leq b$ if and only if $x \leq A^{*} \otimes^{\prime} b$ where $\otimes^{\prime}$ is a dual operation. 
Corollary 3. If $A \in R^{m \times n}, b \in R^{m}$ and $c \in R^{n}$ then

(a) $A^{*} \otimes^{\prime} b$ is the greatest solution to $A \otimes x \leq b$, that is:

$A \otimes\left(A^{*} \otimes^{\prime} b\right) \leq b$

(b) $A \otimes x=b$ has a solution if and only if $\bar{x}$ is a solution and

(c) $A \otimes\left(A^{*} \otimes^{\prime}(A \otimes c)\right)=A \otimes c$

where $\bar{x}=A^{*} \otimes^{\prime} b$ is the principal solution to $A \otimes x \leq b$ and $A \otimes x=b$.

\section{Max-Plus Formulation of Genetic Algorithm}

The basic concept of GAs is to simulate processes in a system based on the principle of natural selection. The technique begins with with a set of possible solutions (chromosomes) called population. Usually, evolution through selection, mutation, and recombination of these solutions leads to a better desired solution, which, hopefully converges to the sought optimal solution. These solutions (offspring) are selected based on a fitness function.

GA's are usually applied to discrete event systems. In general, such systems are nonlinear in conventional algebra. Reformulation of such systems using max-plus algebra reduces them to linear systems. Consider the following optimization problem:

$$
\begin{gathered}
\operatorname{Max}\left(f\left(x_{i}\right), i=1, \ldots, n\right) \\
\text { subject to: } V_{i}+W_{i}=B_{i},
\end{gathered}
$$

where $V_{i}, W_{i}, B_{i}$ are vectors. By Theorem 4 and Corollary 3 , the solution to problem (8) exists if it can be converted to max-plus form. If the system above can be characterized as a discrete event system in which only synchronization and no concurrency or choice occurs [6], then the system can be converted into a max-plus linear system, even though the original system may be a nonlinear programing problem.

The max-plus algebra formulation for the above system (8) becomes:

$$
V_{i} \bigotimes_{i=1}^{n} W_{i} \bigoplus_{i=1}^{n} f\left(x_{i}\right)
$$

By Theorems 1, 4, and Corollary 3, problem (9) has a solution. 


\subsection{Max-plus GA Steps}

Consider the initial population $P$ of chromosomes which are selected randomly based on the requirements imposed on the solution, then

$$
P=\left[\begin{array}{c}
x_{1} \\
x_{2} \\
\vdots \\
x_{n}
\end{array}\right]
$$

where $x(i) \in \Omega$ is selected randomly.

Note that for any GA, a chromosome representation is needed to describe each individual in the population of interest. The following section describes the max-plus formulation for GAs.

\section{Evaluation Process}

Individual chromosomal strengths of the population are determined by evaluating their max-plus fitness using the function $f$. That is:

$$
\left[\begin{array}{c}
x_{1} \\
x_{2} \\
\vdots \\
x_{n}
\end{array}\right] \longrightarrow\left[\begin{array}{c}
f\left(x_{1}\right) \\
f\left(x_{2}\right) \\
\vdots \\
f\left(x_{n}\right)
\end{array}\right]
$$

where the fittest individual is determined by the equation below:

$$
\begin{aligned}
f\left(x_{1}\right) \oplus f\left(x_{2}\right) \oplus \ldots \oplus f\left(x_{n}\right) & =\max \left(f\left(x_{1}\right), f\left(x_{2}\right), \ldots, f\left(x_{n}\right)\right) \\
& =\max _{i \in n} f\left(x_{i}\right)
\end{aligned}
$$

and $x(i)=x_{\max } \in \Omega$ is the fittest chromosome to survive in the next generation.

\section{Selection Process}

A probabilistic selection is performed based on the the fitness of the individual such that the better chromosomes have more advantage of being selected. There are several selection schemes [12]. In "Roulette wheel" for example, the probability of each individual $P_{i}$ is given by:

$$
P(\text { Chromosome } i \text { is chosen })=\frac{f\left(x_{j}\right)}{\sum_{i}^{n} f\left(x_{i}\right)}
$$


where $f\left(x_{i}\right)$ is fitness of chromosome $i$. The distribution of a randomly chosen chromosome (in population) after selection is given by:

$$
x(n)=\frac{\sigma\left(f\left(x_{j}\right) x_{j}(n)\right.}{\sum_{i}^{n} \sigma\left(f\left(x_{i}\right)\right) x_{i}(n)}
$$

where $\sigma$ is the selection function.

A new population is obtained where $y_{i}=x_{j}$, that is:

$$
P^{\prime}=\left[\begin{array}{c}
y_{1} \\
y_{2} \\
\vdots \\
y_{n}
\end{array}\right]
$$

All of the chromosomes of $P^{\prime}$ are those of $P$ and the expectation of the number of occurrences of any chromosome of $P$ in $P^{\prime}$ is proportional to the number of occurrences of that chromosome in $P$ multiplied by the chromosomes fitness value, that is $E\left(x_{i}\right)=x_{i} f\left(x_{i}\right)$.

3. Reproduction Process

The chromosomes of $P^{\prime}$ are grouped for mating according to a new probabilistic rule and transformation $\mathcal{T}$. The new groups are:

$$
\mathbf{Q}_{1}=\left[\begin{array}{c}
y_{i_{1}^{1}} \\
y_{i_{2}^{1}} \\
\vdots \\
y_{i_{q_{1}}^{1}}
\end{array}\right] \quad \mathbf{Q}_{2}=\left[\begin{array}{c}
y_{i_{1}^{2}} \\
y_{i_{2}^{2}} \\
\vdots \\
y_{i_{q_{2}}^{2}}
\end{array}\right] \ldots \mathbf{Q}_{j}=\left[\begin{array}{c}
y_{i_{1}^{j}} \\
y_{i_{2}^{j}} \\
\vdots \\
y_{i_{q_{j}}^{j}}
\end{array}\right] \ldots
$$

Applying the crossover transformation process we have:

$$
\mathbf{Q}^{\mathcal{T}}=\left[\begin{array}{c}
\mathcal{T}_{1}\left(y_{i_{1}^{j}}, y_{i_{2}^{j}}, \ldots, y_{i_{q_{j}}^{j}}\right) \\
\mathcal{T}_{2}\left(y_{i_{1}^{j}}, y_{i_{2}^{j}}, \ldots, y_{i_{q_{j}}^{j}}\right) \\
\vdots \\
\mathcal{T}_{q_{j}}\left(y_{i_{1}^{j}}, y_{i_{2}^{j}}^{j}, \ldots, y_{i_{q_{j}}^{j}}\right)
\end{array}\right]
$$

This results in the next generation population of chromosomes

$$
P^{\prime \prime}=\left[\begin{array}{c}
z_{1} \\
z_{2} \\
\vdots \\
z_{n}
\end{array}\right]
$$


where $\mathcal{T}_{l}\left(y_{i_{1}^{j}}, y_{i_{2}^{j}}, \ldots, y_{i_{q_{j}}^{j}}\right)=z_{l}, l=1, \ldots, n$. $P^{\prime \prime}$ then undergoes a mutation process. Mutation serves as a supporting operator for restoring lost genetic traits. Thus, with a very small probability we replace $z_{i}$ with $w_{i}$ for some randomly chosen element and this gives a new population

$$
P^{\prime \prime \prime}=\left[\begin{array}{c}
w_{1} \\
w_{2} \\
\vdots \\
w_{n}
\end{array}\right]
$$

If we are not satisfied with the solution, we start all over with $P^{\prime \prime \prime}$ as the initial population and the cycle is repeated a certain number of times depending on the fitness function.

\subsection{Max-plus Search Space and Fitness Function}

To formulate the max-plus model for genetic algorithm, we consider the space $\Omega=\left\{x_{1}, x_{2}, \ldots, x_{n}\right\}$ as a finite set of possible solutions of the GA known as the search space or solution space. For each $x_{i} \in \Omega, 1 \leq i \leq n$ where the $x_{i}$ 's are chromosomes or individuals. We claim that the solution space is an idempotent semi-ring.

Theorem 5. The GA search space is a max-plus commutative idempotent semi-ring.

Proof. We note that $\oplus$ is associative in the search space, that is for all chromosomes $x_{1}, x_{2}, x_{3} \in \Omega, x_{1} \oplus\left(x_{2} \oplus x_{3}\right)=\left(x_{1} \oplus x_{2}\right) \oplus x_{3}$ and commutative with zero element $\varepsilon$, thus $x_{1} \oplus \varepsilon=\varepsilon \oplus x_{1}=x_{1}$. Again we see that $\otimes$ is associative, distributive over $\oplus$ and has unit element $e$ since for all elements $x_{1}, x_{2}, x_{3} \in \Omega, x_{1} \otimes\left(x_{2} \oplus x_{3}\right)=\left(x_{1} \otimes x_{2}\right) \oplus\left(x_{1} \otimes x_{3}\right)$ and $x_{1} \otimes e=e x_{1} \otimes x_{1}=x_{1}$. Next, $\varepsilon$ is absorbing for $\otimes$ since $x_{1} \otimes \varepsilon=\varepsilon \otimes x_{1}=\varepsilon$ for all $x_{1} \in \Omega$. We know that $\otimes$ is commutative for all chromosomes $x_{1}, x_{2} \in \Omega$ since $x_{1} \otimes x_{2}=x_{2} \otimes x_{1}$. Finally, $\oplus$ is idempotent, and so for all $x_{1} \in \Omega, x_{1} \oplus x_{1}=x_{1}$. Hence the GA search space is a max-plus commutative idempotent semi-ring.

We now consider the function given by $f: \Omega \rightarrow \mathbb{R}_{\max }$ defined on some domain, then the goal of the function $f$ is to evaluate the maximum functional value determined by a chromosome in the search space, that is $x_{i} \in \Omega$ such that $f\left(x_{i}\right) \rightarrow f_{\max }\left(x_{i}\right)$ for all $1 \leq i \leq n$. The individual chromosome $x_{i} \in \Omega$ which gives the maximum fitness is the fittest chromosome to survive in the 
next generation. That is,

$$
\begin{aligned}
f\left(x_{1}\right) \oplus f\left(x_{2}\right) \oplus \ldots \oplus f\left(x_{n}\right) & =\max \left(f\left(x_{1}\right), f\left(x_{2}\right), \ldots, f\left(x_{n}\right)\right) \\
& =\max _{i \in n} f\left(x_{i}\right) \\
& =\arg \max _{i \in n} f\left(x_{i}\right)
\end{aligned}
$$

Thus given a search space $\Omega=\left\{x_{1}, x_{2}, \ldots, x_{n}\right\}$ and a function $f: \Omega \rightarrow \mathbb{R}_{\max }$, we have $f_{\text {max }}=f\left(x_{\max }\right)=\arg \max _{i \in n} f\left(x_{i}\right)$ where $x_{\text {max }}$ is the fittest chromosome and $f_{\max }$ is the maximum fitness.

\subsection{Max-Plus Formulation for Population Dynamics in Genetic Algorithms}

We use population dynamics model to illustrate the theoretical framework outlined in Sections 3.1 and 3.2. Given any population $X$ then, the population is a vector of chromosomes $x^{(k)}$ given by:

$$
\mathbf{x}^{(k)}=\left[\begin{array}{c}
x_{1}^{(k)} \\
x_{2}^{(k)} \\
\vdots \\
x_{n}^{(k)}
\end{array}\right]
$$

where $x_{i}^{(k)}$ is the number of chromosomes at generation $k$.

The probability that a member of the $k^{\text {th }}$ population will survive to become a member of the $(k+1)^{t h}$ population is $P_{k}$, where $0 \leq P_{k} \leq 1, k=1,2, \ldots, n-1$. Let $b_{k}$ be the reproduction rate, then $b_{k} \geq 0$ for $k=1,2, \ldots, n$. Then these numbers can be written in matrix form as follows:

$$
T=\left[\begin{array}{cccccc}
b_{1} & b_{2} & b_{3} & \cdots & b_{n-1} & b_{n} \\
P_{1} & 0 & 0 & \cdots & 0 & 0 \\
0 & P_{2} & 0 & \cdots & 0 & 0 \\
\vdots & \vdots & \vdots & \cdots & \vdots & \vdots \\
0 & 0 & 0 & \cdots & P_{n-1} & 0
\end{array}\right]
$$

where $T$ is the projection matrix. Operating $T$ on the population vector $\mathbf{x}^{(k)}$ produces the population vector for the next generation. This procedure is represented as a recursive max-plus equation of the form

$$
\mathbf{x}^{(k+1)}=T \otimes \mathbf{x}^{(k)}
$$


which generate the following system of linear equations:

$$
\left[\begin{array}{c}
x_{1}^{(k+1)} \\
x_{2}^{(k+1)} \\
\vdots \\
x_{n}^{(k+1)}
\end{array}\right]=\left[\begin{array}{cccccc}
b_{1} & b_{2} & b_{3} & \cdots & b_{n-1} & b_{n} \\
P_{1} & 0 & 0 & \cdots & 0 & 0 \\
0 & P_{2} & 0 & \cdots & 0 & 0 \\
\vdots & \vdots & \vdots & \cdots & \vdots & \vdots \\
0 & 0 & 0 & \cdots & P_{n-1} & 0
\end{array}\right] \otimes\left[\begin{array}{c}
x_{1}^{(k)} \\
x_{2}^{(k)} \\
\vdots \\
x_{n}^{(k)}
\end{array}\right]
$$

which can be expanded as the following systems of linear max-plus equations

$$
\begin{aligned}
x_{1}^{(k+1)} & =\left(b_{1} \otimes x_{1}^{(k)}\right) \oplus\left(b_{2} \otimes x_{2}^{(k)}\right) \oplus \ldots \oplus\left(b_{n} \otimes x_{n}^{(k)}\right) \\
& =\max \left[\left(b_{1}+x_{1}^{(k)}\right),\left(b_{2}+x_{2}^{(k)}\right), \ldots,\left(b_{n}+x_{n}^{(k)}\right)\right] \\
x_{2}^{(k+1)} & =\left(P_{1} \otimes x_{1}^{(k)}\right) \oplus\left(0 \otimes x_{2}^{(k)}\right) \oplus \ldots \oplus\left(0 \otimes x_{n}^{(k)}\right) \\
& =\max \left[\left(P_{1}+x_{1}^{(k)}\right),\left(0+x_{2}^{(k)}\right), \ldots,\left(0+x_{n}^{(k)}\right)\right] \\
& \vdots \\
x_{n}^{(k+1)} & =\left(0 \otimes x_{1}^{(k)}\right) \oplus\left(0 \otimes x_{2}^{(k)}\right) \oplus \ldots \oplus\left(P_{n-1} \otimes x_{n-1}^{(k)}\right) \oplus\left(0 \otimes x_{n}^{(k)}\right) \\
& =\max \left[x_{1}^{(k)}, x_{2}^{(k)}, \ldots, P_{n-1}+x_{n-1}^{(k)}, x_{n}^{(k)}\right]
\end{aligned}
$$

The population distribution at any generation $k$ is therefore given by:

$$
\begin{aligned}
\mathbf{x}^{(k+1)} & =T \otimes x^{(k)} \\
& =T \otimes\left(T^{\otimes(k)} \otimes x(0)\right) \\
& =\left(T \otimes T^{\otimes(k)}\right) \otimes x(0) \\
& =\overbrace{T \otimes T \otimes \ldots \otimes T}^{\text {times }} \otimes x(0) \\
& =T^{\otimes k} \otimes x(0)
\end{aligned}
$$

We note that the numerical evaluation of $T^{\otimes k}$ is $k \times T$ in conventional algebra. Thus, if we know the initial generation vector

$$
\mathbf{x}^{(0)}=\left[\begin{array}{c}
x_{1}^{(0)} \\
x_{2}^{(0)} \\
\vdots \\
x_{n}^{(0)}
\end{array}\right]
$$

We can determine the next vector by multiplying $\mathbf{x}^{(0)}$ by an appropriate power of the projection matrix. Alternatively, equation (5) can be interpreted in the 
max-plus algebra as follows:

$$
\begin{aligned}
\mathbf{x}^{(n+1)} & =T \otimes \mathbf{x}^{(n)} \\
& =\left(T_{i 1} \otimes x_{1}^{(n)}\right) \oplus \ldots \oplus\left(T_{i n} \otimes x_{n}^{(n)}\right) \\
& =\bigoplus_{j=1}^{k}\left(T_{i j} \otimes x_{j}^{(n)}\right) \\
& =\max _{1 \leq j \leq k}\left(T_{i j}+x_{j}^{(n)}\right)
\end{aligned}
$$

The subsequent generations is obtained by the max-plus multiplication of the projection matrix $T$ by an initial matrix from the search space related to the population dynamics of the problem. Hence $\mathbf{x}^{(n+1)}=\max _{1 \leq j \leq k}\left(T_{i j}+x_{j}^{(n)}\right)$ is the fittest population to begin the next cycle.

\subsection{Convergence of the Max-Plus GA}

To establish convergence, we expect that the algorithm tends to an optimum. We note in general that the search space in GA is simply a set without any norm or distance measure. Therefore we cannot anticipate convergence criteria saying that $x^{n+1}$ tends to a limiting value (optimum) as $n$ tends to infinity. Instead, we require that the best solution would be a measure of diversity of the chromosomes. To see this, we look at the max-plus asymptotic behavior of $x(k)$ quantitatively. We consider $\{x(k): k \in \mathbb{N}\}$ as a sequence in the max-plus algebra and assume that for all $j \in n$ the quantity $\eta_{j}$, defined by

$$
\eta_{j}=\lim _{k \rightarrow \infty} \frac{x_{j}^{(k)}}{k}
$$

exists, where

$$
x_{i}^{(k+1)}=\max _{1 \leq i \leq n}\left(A_{i j}+x_{j}^{(k)}\right), \forall 1 \leq i \leq n
$$

Then the vector $\eta=\left(\eta_{1}, \eta_{2}, \ldots, \eta_{n}\right)^{T}$ is called the cycle time vector of the sequence $\mathbf{x}^{(k)}$. If all $\eta_{j}^{\prime} s$ have the same value, this value is called the asymptotic growth rate of the sequence $\mathbf{x}(k)$. In this case the sum of deviations among individuals become smaller and smaller and there is lack of improvement in the best solution over a specified number of generations determined by the cycle time vector. We apply the max-plus asymptotic growth rate of the sequence $\mathbf{x}(n)$ to terminate the genetic algorithm. We note that equation (8) is a linear system in the max-plus semi-ring $\mathbf{x}^{(n+1)}=A \otimes \mathbf{x}^{(n)}$ and apply the population 
convergence criteria of GA's and use a measure of diversity in the current population to predict the solution to the problem. This may not guarantee a global maximum. The solution may turn out to be a local maximum. It is possible to combine the GA model with Simulated Annealing, for example, to prevent settling for a local maximum [14].

\subsection{The Max-Plus GA Complete Model}

We present the complete max-plus GA formulation and specify the interaction between the projection matrix and the generation vectors. The fitness function is evaluated by the max-plus equation

$$
\begin{aligned}
f\left(x_{1}\right) \oplus f\left(x_{2}\right) \oplus \ldots \oplus f\left(x_{n}\right) & =\max \left(f\left(x_{1}\right), f\left(x_{2}\right), \ldots, f\left(x_{n}\right)\right) \\
& =\max _{i \in n} f\left(x_{i}\right) \\
& =\arg \max _{i \in n} f\left(x_{i}\right) \text { where } x_{i} \in \Omega
\end{aligned}
$$

The population dynamics for the generation is given by the max-plus equations

$$
\begin{aligned}
\mathbf{x}^{(k+1)} & =T \otimes x^{(k)} \\
& =T \otimes\left(T^{\otimes(k)} \otimes x(0)\right) \\
& =\left(T \otimes T^{\otimes(k)}\right) \otimes x(0) \\
& =\overbrace{T \otimes T \otimes \ldots \otimes T}^{\text {ktimes }} \otimes x(0) \\
& =T^{\otimes k} \otimes x(0)
\end{aligned}
$$

Therefore for a given $x(0)$, the sequence $x(k), k=1,2, \ldots$ generated by the max-plus equation $x^{(k+1)}=T \otimes x^{(k)}, k \geq 0$ yield a sequence of increasingly better solutions indicated by the iterates $x(k)$ with the projection matrix. This is shown by the function $\max _{1 \leq j \leq k}\left(T_{i j}+x_{j}(n)\right)$ below, which shows the maximum of the interaction between the projection matrix and the current population vector and in max-plus, this is the fittest population to begin the next generation.

$$
\begin{aligned}
x(n+1) & =T \otimes x(n) \\
& =\left(T_{i 1} \otimes x_{1}(n)\right) \oplus \ldots \oplus\left(T_{i n} \otimes x_{n}(n)\right) \\
& =\bigoplus_{j=1}^{k}\left(T_{i j} \otimes x_{j}(n)\right)
\end{aligned}
$$




$$
=\max _{1 \leq j \leq k}\left(T_{i j}+x_{j}(n)\right)
$$

The equation below actually plays a larger row in max-plus algebra representation of convergence of the GA that is,

$$
\begin{gathered}
T \otimes x(n)=\lambda \otimes x(n) \text { and } \\
\lim _{k \rightarrow \infty} \frac{x_{j}(k)}{k}=\eta_{j}
\end{gathered}
$$

where $\eta_{j}^{\prime} s$ are the cycle-time vectors of the sequence $x(k)$ and

$$
x_{i}(k)=\max _{1 \leq i \leq n}\left(A_{i j}+x_{j}(k-1)\right), \forall 1 \leq i \leq n
$$

\section{Conclusion}

We have reformulated GA optimization method in a more effective manner. Our theoretical approach obtains the optimal solution to a constrained optimization problem using GA by comparing the analytical determination of the fitness function using max-plus algebra [15].

One of the disadvantages of GAs is that the fitness function may lead to GA solving a different problem entirely from the originally intended one or GA can converge to a local solution, instead of the sought after global solution. Our max-plus algebra formulation ensures that maximum fitness function is obtained by summing the functional values of all chromosomes in the search space $\Omega$, that is $\max _{i \in n} f\left(x_{i}\right)$ for which $x_{i}=x_{\max }$ is the fittest chromosomes. Many of the equations that are encountered in real life to which GA's are applied are nonlinear in conventional algebra but become linear in the maxplus algebra. This leads to more convenient solution in terms of computational cost.

Another issue with GAs is setting the correct population size. If the population count is too small, the investigation may cover too little of the search space to find the optimum solution. The max-plus formulation does not restrict the number of chromosomes in the population. Finally, the use of the stable growth max-plus equation $\lambda^{(k+1)}=\lambda \otimes x^{(k)}$ normalizes the GA system and makes it stable with constant population. Hence the lengthy and complicated trial-and-error procedure whose purpose is to adjust the parameters of the GA is avoided. 


\section{References}

[1] M. Akian, G. Cohen, S. Gaubert, J. P. Quadrat, and M. Viot, MaxPlus algebra and applications to system theory and optimal control, Proceedings of International of Mathematicians, Zurich, Switzerland, 1994, Birkhauser, 1502-1511, 1995.

[2] M. Akian, J. P.Quadrat, M. Viot, Duality between probability and optimization, in Idem-potency(Gunawardena ed.), Cambridge Univ. Press, 1998.

[3] X. Allamigeon, S. Gaubert, and E. Goubault, Inferring min and max invariants using max-plus polyhedra. In proceedings of the 15th International Static Analysis Symposium (SAS08), Springer LNCS 5079 (2008), 189204.

[4] A. Aminu, On the solvability of homogeneous two-sided systems in maxalgebra, NNTDM 162 (2010), 5-15.

[5] T.J.J van den Boom, B. Schutter, G. Schullerus, and V. Krebs, Adaptive model predictive control for max-plus-linear discrete event input-output systems, IEE Proceedings-Control Theory and Applications, 151 (2004), 339-346.

[6] T.J.J van den Boom and B. De Schutter, 2006, Dynamic railway network management using switching max-plus-linear models, Proceedings of the 11th IFAC Symposium on Control in Transportation Systems, Delft, Netherlands, 343-348.

[7] T.J.J van den Boom and B. De Schutter, 2004, Modelling and control of discrete event systems using switching max-plus linear systems. Proceedings of the 7th International Workshop on Discrete Event Systems (WODES '04), Reims, France 115-120.

[8] P. Butkovic and K. Po Tam, On some properties of the image set of a max-linear mapping, Contemporary Mathematics, 495 (2009), 115-126.

[9] M. Gondran, and M. Minoux, Linear algebra in diodids: A survey of recent results, Annals of Discrete Mathematics, 19 (1984), 130-160.

[10] J. Edmonds, R. M. Karp, Theoretical improvements in algorithmic efficiency for network flow problems, Journal of Association for Computing Machinery 19 (1972), 248-264. 
[11] L. Elsener and P. van den Driessche, On the power method in max algebra, Linear Algebra and its Applications, 1999 302-303 17-32.

[12] D.Goldberg, Genetic Algorithms in Search, Optimization and Machine Learning, Addison-Wesley, USA (1989).

[13] H. Myskovsa, Solvability concepts for interval systems in Max-Plus Algebra, Journal of Applied Mathematics, 3 (2010), 225-236.

[14] H. Nobuhara and C. Han, Evolutionary computation schemes based on max-plus algebra and their application to image processing, International Symposium on Intelligent Signal Processing and Communication Systems (ISPAC 2006), Japan (2006).

[15] S. Smolorz and B.Wagner, Self-organized distribution tasks inside an autonomous mobile robotic system, 6th International Conference on Digital Ecosytems Technology (DEST), (2012) 1-4.

[16] H. Tom āškova, Max-plus Algebra and its applications in spreading of information, Advances in Mathematical and Computational Methods, 4 (2012), 188-191. 